Check for updates

Cite this: RSC Adv., 2019, 9, 21525

Received 7th June 2019

Accepted 5th July 2019

DOI: $10.1039 / c 9 r a 04282 b$

rsc.li/rsc-advances

\title{
Copper-catalyzed synthesis of phenol and diaryl ether derivatives via hydroxylation of diaryliodoniums $\uparrow$
}

\author{
Lianbao Ye, (D) *a Chao Han, ${ }^{a}$ Peiqi Shi, ${ }^{a}$ Wei Gao ${ }^{a}$ and Wenjie Mei iD b
}

A copper-catalysed hydroxylation of diaryliodoniums to generate phenols and diaryl ethers is reported. This method allows the synthesis of diversely functionalized phenols under mild reaction conditions without the need for a strong inorganic base or an expensive noble-metal catalyst. Significantly, convenient application of diaryliodoniums is demonstrated in the preparation of diaryl ethers in a one-pot operation.

\section{Introduction}

Phenols and their derivatives are ubiquitous constituents for constructing bioactive natural products, agrochemicals and pharmaceuticals and serve as important motifs of polymers as well as novel materials. ${ }^{1}$ Currently, many useful methods have been developed to prepare these phenol moieties by synthetic researchers. ${ }^{2}$ Couplings of aryl halides and nucleophilic hydroxide salts in the presence of palladium or copper complex have been proved to be classical methods to prepare these compounds (Scheme 1a). ${ }^{3 i}$ In addition, hydroxylation of boronic acids (Scheme 1b) and hydrolysis of diazonium salts are also efficient strategies. ${ }^{4}$ Although phenol derivatives are available by different transformations, it is very important to develop novel methodologies with mild reaction conditions, high efficiency and low waste generation.

Diaryliodoniums are air- and moisture-stable versatile electrophilic arylating reagents, which are easily available when iodoarenes are treated with mild oxidants. ${ }^{5}$ They have been utilized to arylate a broad scope of nucleophiles in recent years, including aryl/alkyl amines, ${ }^{6}$ phenols, ${ }^{7}$ aliphatic alcohol and benzoic acid, which afford the corresponding aromatic compounds. ${ }^{8}$ Despite this achievement, the use of strong inorganic bases or organic bases is adverse to reaction, especially for basic-sensitive functional groups. ${ }^{9}$ Recently, We and other groups reported mild and rapid transformations of cyclic hypervalent iodoniums into oxygen-bridged polycyclic heteroarenes and dibenzofuran derivatives. ${ }^{10}$ Owing to these previous work, we envisage a strategy for the synthesis of phenols via the

${ }^{a}$ School of Pharmacy, Guangdong Pharmaceutical University, Guangzhou 510006, Guangdong, China. E-mail: yelb7909@163.com; Tel: +86-20-39352139

${ }^{b}$ Guangdong Province Engineering Technology Center for Molecular Probes \& Biomedical Imaging, Guangdong Pharmaceutical University, Guangzhou 510006, Guangdong, China

$\dagger$ Electronic supplementary information (ESI) available. See DOI: 10.1039/c9ra04282b hydroxylation of diaryl iodoniums. In terms of the oxygen donor, waster is the most environmentally friendly oxygen source. ${ }^{11}$ The arylation of diaryliodoniums with waster as the nucleophile is believed to be a valuable access to phenols. In this study, we develop a one-pot reaction for the synthesis of a wide range of phenols and diaryl ethers from diaryliodoniums. This versatile method operates under mild reaction conditions, with inexpensive $\mathrm{CuCl}$ as the catalyst (Scheme 1c).

\section{Results and discussion}

At the outset of our studies, the hydroxylation of diaryliodoniums was conducted by the exposure substrate 1a to $\mathrm{Cu}(\mathrm{OAc})_{2}$ (0.1 equiv.), $\mathrm{Na}_{2} \mathrm{CO}_{3}$ (2.0 equiv.), $\mathrm{H}_{2} \mathrm{O}$ (5.0 equiv.) in $\mathrm{DMF}$ at $40{ }^{\circ} \mathrm{C}$ for 2 hours, leading to the desired product $2 \mathrm{a}$ in a moderate yield (entry 1). Encouraged by this result, we subsequently screened other copper catalysts (entry 2-5),

a) Hydroxylation of halides to synthesize phenol

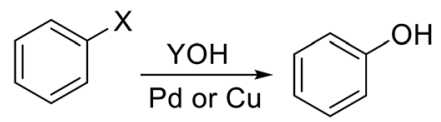

$\mathrm{X}=\mathrm{I}, \mathrm{Br}, \mathrm{Cl}$

$\mathrm{Y}=\mathrm{Li}, \mathrm{Na}, \mathrm{K}, \mathrm{Cs}$

b) Hydroxylation of boronic acids to synthesize phenol

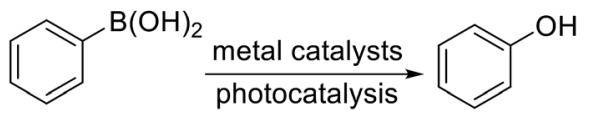

c) This work

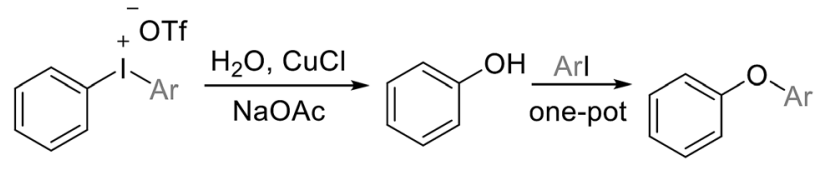

Scheme 1 Selected methods for the synthesis of phenols. 
including $\mathrm{Cu}(\mathrm{OTf})_{2}, \mathrm{CuCl}_{2}, \mathrm{CuSO}_{4}$ and $\mathrm{CuI}$ were inferior to $\mathrm{Cu}(\mathrm{OAc})_{2}$, while $\mathrm{CuCl}$ showed the best efficiency. Compared with DMF, solvents of toluene, DCE, dioxane and MeCN were not helpful for the reaction conversion (entry 6-9). The examine of bases disclosed that mild NaOAc was ideal choice (entry 1014). The yields reduced when the reaction was performed in shortened time (entry 15-16). It was observed that only 39\% 2a was yielded at rt (entry 17), which indicated that slight heating was needed for the diaryliodoniums reactivity. Further study revealed that it is not fruitful without the addition of water (entry 18), and even no targeted product was isolated in the absence of CuCl (entry 19) (Table 1).

With optimized reaction conditions in hand, diversely functionalized diaryliodoniums were subjected to the established reaction conditions to explore the efficiency and scope of this copper-catalyzed hydroxylation reaction. As summarized in Scheme 2, this protocol afforded phenols bearing electron-rich, electron-poor, or sterically bulky substituted groups in good yields (2a-2l). Electron-neutral phenols were delivered in excellent yields (2a-2c). In terms of electron-deficient substrates, para-, and meta-substituted diaryliodoniums containing $-\mathrm{F},-\mathrm{OCF}_{3},-\mathrm{CF}_{3}$ and $-\mathrm{CO}_{2} \mathrm{Me}$ were compatible to produce phenols $2 \mathbf{d}-\mathbf{2 g}$ in the yield of $56-87 \%$. The reaction also furnished the electron-rich product efficiently $(2 \mathbf{h})$. Notably, 1-naphthol was available in a $74 \%$ yield (2i). It is

Table 1 Optimization of the hydroxylation of diaryliodoniums. Reaction conditions: $1 \mathrm{a}(0.1 \mathrm{mmol})$, catalyst (10 mol\%), base (2 equiv.), $\mathrm{H}_{2} \mathrm{O}$ (5 equiv.), solvent $(0.5 \mathrm{~mL}), \mathrm{Ar}, 2 \mathrm{~h} .{ }^{\mathrm{a}} 1.5 \mathrm{~h},{ }^{\mathrm{b}} 1 \mathrm{~h},{ }^{\mathrm{c}} 12 \mathrm{~h},{ }^{\mathrm{d}} \mathrm{H}_{2} \mathrm{O}$ was not added

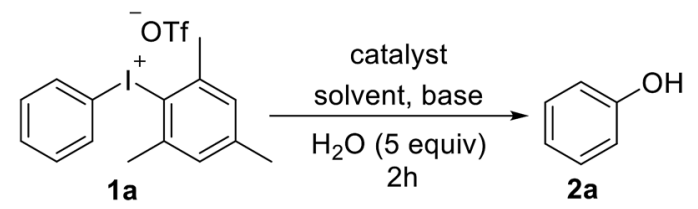

\begin{tabular}{|c|c|c|c|c|c|}
\hline Entry & Catalysis & Base & Solvent & $\begin{array}{l}T \\
\left({ }^{\circ} \mathrm{C}\right)\end{array}$ & Yield (\%) \\
\hline 1 & $\mathrm{Cu}(\mathrm{OAc})_{2}$ & $\mathrm{Na}_{2} \mathrm{CO}_{3}$ & DMF & 40 & 57 \\
\hline 2 & $\mathrm{Cu}(\mathrm{OTf})_{2}$ & $\mathrm{Na}_{2} \mathrm{CO}_{3}$ & DMF & 40 & 0 \\
\hline 3 & $\mathrm{CuSO}_{4}$ & $\mathrm{Na}_{2} \mathrm{CO}_{3}$ & DMF & 40 & 0 \\
\hline 4 & $\mathrm{CuI}$ & $\mathrm{Na}_{2} \mathrm{CO}_{3}$ & DMF & 40 & 50 \\
\hline 5 & $\mathrm{CuCl}$ & $\mathrm{Na}_{2} \mathrm{CO}_{3}$ & DMF & 40 & 85 \\
\hline 6 & $\mathrm{CuCl}$ & $\mathrm{Na}_{2} \mathrm{CO}_{3}$ & Toluene & 40 & 10 \\
\hline 7 & $\mathrm{CuCl}$ & $\mathrm{Na}_{2} \mathrm{CO}_{3}$ & DCE & 40 & 21 \\
\hline 8 & $\mathrm{CuCl}$ & $\mathrm{Na}_{2} \mathrm{CO}_{3}$ & Dioxane & 40 & 0 \\
\hline 9 & $\mathrm{CuCl}$ & $\mathrm{Na}_{2} \mathrm{CO}_{3}$ & MeCN & 40 & 42 \\
\hline 10 & $\mathrm{CuCl}$ & $\mathrm{Et}_{3} \mathrm{~N}$ & DMF & 40 & 77 \\
\hline 11 & $\mathrm{CuCl}$ & $\mathrm{K}_{2} \mathrm{CO}_{3}$ & DMF & 40 & 85 \\
\hline 12 & $\mathrm{CuCl}$ & NaOAc & DMF & 40 & 92 \\
\hline 13 & $\mathrm{CuCl}$ & $\mathrm{K}_{3} \mathrm{PO}_{4}$ & DMF & 40 & 14 \\
\hline 14 & $\mathrm{CuCl}$ & $\mathrm{NaOH}$ & DMF & 40 & 0 \\
\hline 15 & $\mathrm{CuCl}$ & NaOAc & DMF & 40 & $86^{a}$ \\
\hline 16 & $\mathrm{CuCl}$ & NaOAc & DMF & 40 & $79^{b}$ \\
\hline 17 & $\mathrm{CuCl}$ & NaOAc & DMF & $\mathrm{rt}$ & $39\left(83^{c}\right)$ \\
\hline 18 & $\mathrm{CuCl}$ & NaOAc & DMF & 40 & $11^{\mathrm{d}}$ \\
\hline 19 & - & NaOAc & DMF & 40 & 0 \\
\hline
\end{tabular}

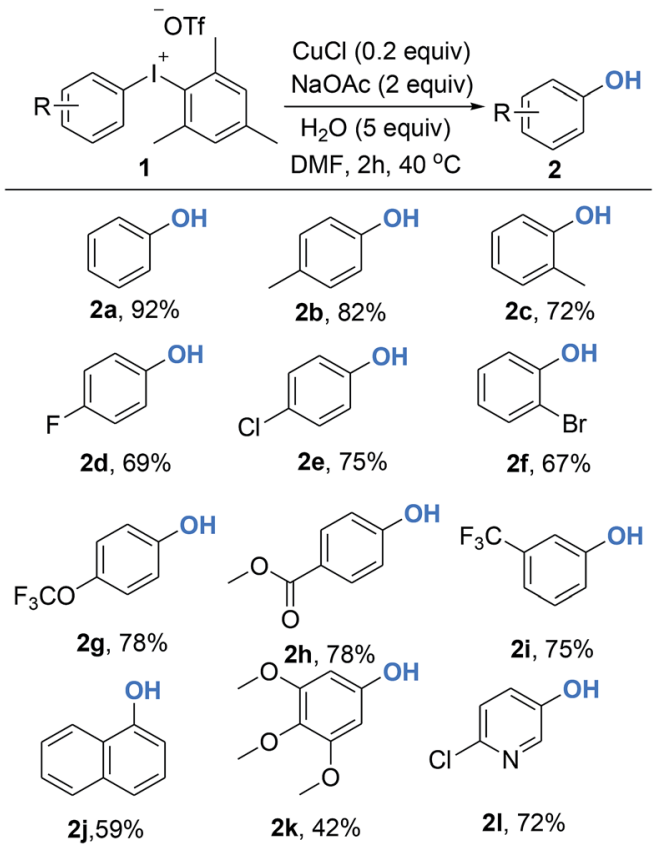

Scheme 2 Copper-catalyzed synthesis of phenols from diaryliodoniums. Reaction conditions: 1 (0.2 mmol), $\mathrm{CuCl}(10 \mathrm{~mol} \%), \mathrm{NaOAc}$ (2 equiv.), $\mathrm{H}_{2} \mathrm{O}$ (5 equiv.), DMF (1.0 mL), $\mathrm{Ar}, 40^{\circ} \mathrm{C}, 2 \mathrm{~h}$.

necessary to emphasize that the reaction conditions were tolerant of ortho-functional groups such as methyl and bromo to give $2 \mathbf{j}$ and $\mathbf{2 k}$. Hydroxylated heteroarenes are prevalent bioactive intermediates in medicinal chemistry. To our surprise, it was demonstrated that our strategies were suitable for the synthesis of hydroxylated pyridine $\mathbf{2 l}$.

Diaryl ethers are significant building blocks and have found wide applications in the preparation of natural products and synthetic compounds with various biological properties and functions. ${ }^{12}$ Although diaryliodoniums have been extensively investigated as versatile arylating reagents in recent years, their poor atom economy limits further applications, as one equivalent of iodobenzene was generated as a waste in most of common reactions. ${ }^{13}$ Until recently, some examples were reported to overcome the drawback by designing one-pot, tandem reactions that can use the aryl iodide side product in a second arylation process in situ. ${ }^{14}$ Inspired by this domino arylating strategy, it is appealing to synthesize diaryl ethers directly, which represents a feasibility of using diaryliodoniums in an economic way. As shown in Scheme 3, the access to various aromatic ethers was realized by the addition of $\mathrm{Cs}_{2} \mathrm{CO}_{3}$ and dimethylglycine at $130{ }^{\circ} \mathrm{C}$. As expected, symmetric diaryl ethers substituted by both electron-donating and electronwithdrawing groups formed smoothly $(\mathbf{4 a - 4 g})$. It is notable that the intact chloro (4e) or bromo (4f) moieties allow potential modification of polymer materials. Besides, unsymmetric diaryl ethers were synthesized efficiently in our method $(\mathbf{4 h}-\mathbf{4 i})$. Based on our observation, a copper-catalyzed hydroxylation of linear diaryliodoniums was proposed. Initially, oxidative addition of iodonium 1a to copper(I) would provide copper(III) intermediate A. This electrophilic species can be attacked by $\mathrm{H}_{2} \mathrm{O}$ under basic 

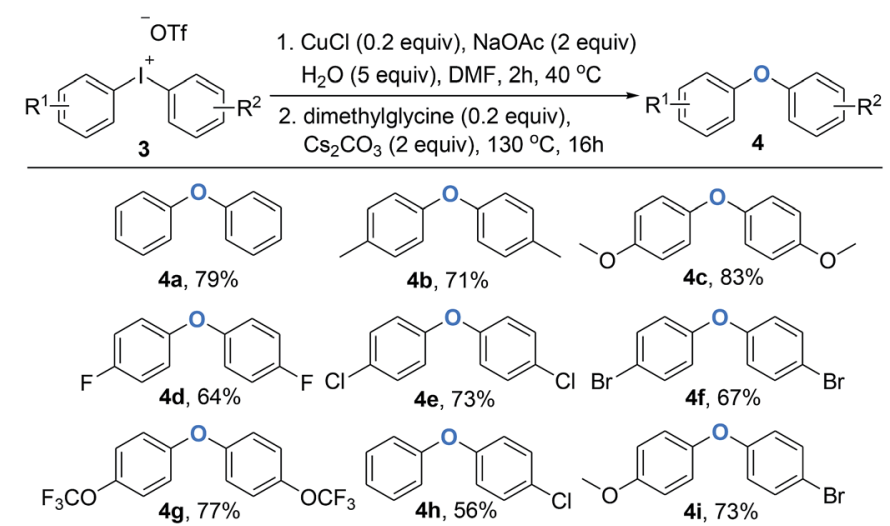

Scheme 3 One-pot synthesis of diaryl ethers from diaryliodoniums. Reaction conditions: (1) $1(0.2 \mathrm{mmol}), \mathrm{CuCl}(10 \mathrm{~mol} \%), \mathrm{NaOAc}(2$ equiv.), $\mathrm{H}_{2} \mathrm{O}$ (5 equiv.), DMF (1.0 mL), $\mathrm{Ar}, 40{ }^{\circ} \mathrm{C}, 2 \mathrm{~h}$; (2) $\mathrm{Cs}_{2} \mathrm{CO}_{3}(2$ equiv.) and dimethylglycine (0.2 equiv.) were added, $\mathrm{Ar}, 130{ }^{\circ} \mathrm{C}, 16 \mathrm{~h}$.

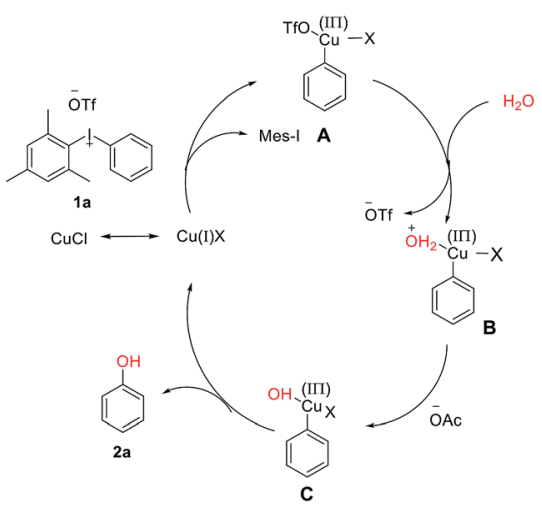

Fig. 1 The proposed mechanism for the hydroxylation of diaryliodoniums.

conditions, and intermediate $\mathrm{B}$ and $\mathrm{C}$ were formed consequently through this process. Finally, a reductive elimination of $\mathrm{C}$ would generate phenol 2a (Fig. 1).

\section{Conclusions}

In summary, we have developed a copper-catalysed hydroxylation diaryliodoniums in a mild reaction conditions. This protocol enables the synthesis of aryl/heteroaryl phenols with various functionalities. Moreover, the one-pot, tandem process to diaryl ethers realizes convenient application of diaryliodoniums. Together with the use of an inexpensive metal catalyst, simple operation and atom economy, featuring the practical value of our protocol. Further study on utilization of this strategy in medicinal chemistry is underway in our lab.

\section{Experimental}

\section{General information}

All solvents were commercially available and were used without further purification unless stated. The chemicals used were either purchased from commercial sources or prepared according to literature procedures for CDPIs. The ${ }^{1} \mathrm{H},{ }^{13} \mathrm{C}$ nuclear magnetic resonance (NMR) spectra were recorded on a Bruker Avance spectrometer 400 at $400 \mathrm{MHz}, 100 \mathrm{MHz}$ respectively. Chemical shifts are given in ppm $(\delta)$ referenced to $\mathrm{CDCl}_{3}$ with 7.26 for ${ }^{1} \mathrm{H}$ and 77.10 for ${ }^{13} \mathrm{C}$, and to $\mathrm{d}_{6}$-DMSO with 2.50 for ${ }^{1} \mathrm{H}$ and 39.5 for ${ }^{13} \mathrm{C}$. In the case of multiplet, the signals are reported as intervals. Signals are abbreviated as follows: $\mathrm{s}$, singlet; d, doublet; t, triplet; q, quartet; m, multiplet. Coupling constants are expressed in hertz. Mass spectra were recorded on a BRUKER VPEXII spectrometer (ESI mode). The progress of the reactions was monitored by thin-layer chromatography on a glass plate coated with silica gel with fluorescent indicator (GF254). Column chromatography was performed on silica gel (200-300 mesh).

\section{General procedure to synthesize phenols (2)}

To a round-bottom flask was added diaryliodonium $(0.2 \mathrm{mmol}$, 1.0 equiv.), $\mathrm{NaOAc}$ (2.0 equiv.), $\mathrm{CuCl}$ ( 0.1 equiv.), 5 (0.1 equiv.), DMF $(1.0 \mathrm{~mL})$. Then the flask was sealed, degassed and recharged with argon. The reaction proceeded at $40{ }^{\circ} \mathrm{C}$ for $2 \mathrm{~h}$ under argon atmosphere. Then, the reaction mixture was extracted with EtOAc, the combined organic layers were washed with $\mathrm{H}_{2} \mathrm{O}$ and brine and dried over anhydrous $\mathrm{Na}_{2} \mathrm{SO}_{4}$, evaporated in vacuo. The residue was purified by column chromatography on a silica gel (PE/EtOAc) to provide compounds 2 .

\section{General procedure to synthesize diaryl ethers (4)}

To a round-bottom flask was added diaryliodonium $(0.2 \mathrm{mmol}$, 1.0 equiv.), $\mathrm{NaOAc}$ (2.0 equiv.), $\mathrm{CuCl}$ ( 0.1 equiv.), 5 ( 0.1 equiv.), DMF $(1.0 \mathrm{~mL})$. Then the flask was sealed, degassed and recharged with argon. The reaction proceeded at $40{ }^{\circ} \mathrm{C}$ for $2 \mathrm{~h}$ under argon atmosphere. Then, $\mathrm{Cs}_{2} \mathrm{CO}_{3}$ (2 equiv.) and dimethylglycine ( 0.2 equiv.) were added to the reaction residue, degassed and recharged with argon again. The reaction proceeded at $130{ }^{\circ} \mathrm{C}$ for $16 \mathrm{~h}$ under argon atmosphere. The reaction mixture was extracted with EtOAc, the combined organic layers were washed with $\mathrm{H}_{2} \mathrm{O}$ and brine and dried over anhydrous $\mathrm{Na}_{2} \mathrm{SO}_{4}$, evaporated in vacuo. The residue was purified by column chromatography on a silica gel (PE/EtOAc) to provide compounds 4.

Phenol (2a). White acicular crystal, yield 94\%. ${ }^{1} \mathrm{H}$ NMR (500 $\mathrm{MHz}$, chloroform-d) $\delta 7.26(\mathrm{t}, J=7.7 \mathrm{~Hz}, 2 \mathrm{H}), 6.95(\mathrm{t}, J=7.4 \mathrm{~Hz}$, $1 \mathrm{H}), 6.85(\mathrm{~d}, J=8.3 \mathrm{~Hz}, 2 \mathrm{H}), 4.29$ (d, $J=101.8 \mathrm{~Hz}, 1 \mathrm{H}) .{ }^{13} \mathrm{C} \mathrm{NMR}$ (125 MHz, chloroform-d) $\delta$ 155.57, 129.82, 120.96, 115.45.

$\boldsymbol{P}$-Cresol (2b). Pale yellow crystal, yield 82\%. Mp: $31-33{ }^{\circ} \mathrm{C}$. ${ }^{1} \mathrm{H}$ NMR (500 MHz, chloroform-d) $\delta 7.04$ (d, $\left.J=7.8 \mathrm{~Hz}, 2 \mathrm{H}\right), 6.74$ $(\mathrm{d}, J=7.7 \mathrm{~Hz}, 2 \mathrm{H}), 2.28$ (s, 3H). ${ }^{13} \mathrm{C} \mathrm{NMR}(125 \mathrm{MHz}$, chloroformd) $\delta 153.32,130.21,130.13,115.19,20.60$.

O-Cresol (2c). Colorless clear liquid, yield 72\%. ${ }^{1} \mathrm{H}$ NMR (400 $\mathrm{MHz}$, chloroform-d) $\delta 7.16-7.05(\mathrm{~m}, 2 \mathrm{H}), 6.85(\mathrm{td}, J=7.4,1.2 \mathrm{~Hz}$, $1 \mathrm{H}), 6.77$ (d, $J=8.0 \mathrm{~Hz}, 1 \mathrm{H}), 2.26$ (s, 3H). ${ }^{13} \mathrm{C}$ NMR (101 MHz, chloroform-d) $\delta 153.92,131.16,127.26,123.85,120.88,115.03$, 15.81 .

4-Fluorophenol (2d). Pale yellow crystal, yield 69\%, mp: 42$44{ }^{\circ} \mathrm{C} .{ }^{1} \mathrm{H}$ NMR (400 MHz, chloroform-d) $\delta$ 7.01-6.88 (m, 2H), 6.85-6.73 (m, 2H), 3.98 (s, 1H). ${ }^{13} \mathrm{C}$ NMR (101 MHz, chloroform- 
d) $\delta 158.63,156.26,151.69,151.67,116.42,116.34,116.24$, 116.01 .

4-Chlorophenol (2e). Clear crystal, yield 75\%. Mp: $42-44{ }^{\circ} \mathrm{C}$. ${ }^{1} \mathrm{H}$ NMR (400 MHz, chloroform-d) $\delta 7.19(\mathrm{~d}, J=8.9 \mathrm{~Hz}, 2 \mathrm{H}), 6.77$ (d, $J=8.9 \mathrm{~Hz}, 2 \mathrm{H}) .{ }^{13} \mathrm{C} \mathrm{NMR}$ (101 MHz, chloroform-d) $\delta 154.61$, 130.00, 126.19, 117.13.

2-Bromophenol (2f). Pale yellow liquid, yield 67\%. ${ }^{1} \mathrm{H}$ NMR (400 MHz, chloroform-d) $\delta 7.47(\mathrm{dd}, J=8.0,1.6 \mathrm{~Hz}, 1 \mathrm{H}), 7.23(\mathrm{td}$, $J=7.7,1.6 \mathrm{~Hz}, 1 \mathrm{H}), 7.03(\mathrm{dd}, J=8.2,1.6 \mathrm{~Hz}, 1 \mathrm{H}), 6.81(\mathrm{td}, J=$ 7.7, $1.6 \mathrm{~Hz}, 1 \mathrm{H}) .{ }^{13} \mathrm{C}$ NMR (101 MHz, chloroform-d) $\delta 152.37$, 132.15, 129.33, 121.96, 116.27, 110.39 .

4-(Trifluoromethoxy)phenol (2g). Brown clear liquid, yield $78 \%$. ${ }^{1} \mathrm{H}$ NMR (400 MHz, chloroform-d) $\delta 7.09(\mathrm{~d}, J=8.7 \mathrm{~Hz}$, $2 \mathrm{H}), 6.82$ (d, $J=8.8 \mathrm{~Hz}, 2 \mathrm{H}) .{ }^{13} \mathrm{C}$ NMR (101 MHz, chloroform-d) $\delta$ 154.33, 142.99, 122.79, 116.31 .

Methyl 4-hydroxybenzoate (2h). White solid, yield 78\%. Mp: 122-124 ${ }^{\circ} \mathrm{C} .{ }^{1} \mathrm{H}$ NMR (400 MHz, chloroform-d) $\delta 7.95(\mathrm{~d}, J=$ $8.6 \mathrm{~Hz}, 2 \mathrm{H}), 6.89(\mathrm{~d}, J=8.6 \mathrm{~Hz}, 2 \mathrm{H}), 6.68(\mathrm{~s}, 1 \mathrm{H}), 3.90(\mathrm{~s}, 3 \mathrm{H}) \cdot{ }^{13} \mathrm{C}$ NMR (101 MHz, chloroform-d) $\delta 167.74,160.55,132.12,122.32$, 115.47, 52.26.

3-(Trifluoromethyl)phenol (2i). Yellow clear liquid, yield 75\%. ${ }^{1} \mathrm{H}$ NMR (500 MHz, chloroform-d) $\delta 7.35(\mathrm{t}, J=8.0 \mathrm{~Hz}, 1 \mathrm{H})$, 7.20 (d, $J=7.7 \mathrm{~Hz}, 1 \mathrm{H}), 7.09$ (s, 1H), 7.01 (dd, $J=8.2,2.4 \mathrm{~Hz}$, 1H). ${ }^{13} \mathrm{C}$ NMR (125 MHz, chloroform-d) $\delta 156.08,130.65,125.31$, 123.14, 119.20, 118.08, 118.05, 118.02, 112.79, 112.76.

Naphthalen-2-ol (2j). White solid, yield 59\%. Mp: 119$121{ }^{\circ} \mathrm{C} .{ }^{1} \mathrm{H}$ NMR (400 MHz, chloroform-d) $\delta 7.77$ (dd, $J=8.2$, $6.4 \mathrm{~Hz}, 2 \mathrm{H}), 7.69$ (d, $J=8.2 \mathrm{~Hz}, 1 \mathrm{H}), 7.46-7.40(\mathrm{~m}, 1 \mathrm{H}), 7.33$ (ddd, $J=8.1,6.9,1.1 \mathrm{~Hz}, 1 \mathrm{H}), 7.15$ (d, $J=2.4 \mathrm{~Hz}, 1 \mathrm{H}), 7.11(\mathrm{dd}, J$ $=8.8,2.5 \mathrm{~Hz}, 1 \mathrm{H}), 4.87$ (s, $1 \mathrm{H}) .{ }^{13} \mathrm{C} \mathrm{NMR}(101 \mathrm{MHz}$, chloroformd) $\delta 153.33,134.60,129.91,128.97,127.81,126.58,126.40$, 123.68, 117.75, 109.51 .

3,4,5-Trimethoxyphenol (2k). Yellowish solid, yield 42\%. Mp: 147-148 ${ }^{\circ} \mathrm{C} .{ }^{1} \mathrm{H}$ NMR (400 MHz, chloroform-d) $\delta 6.09(\mathrm{~s}, 2 \mathrm{H})$, $3.82(\mathrm{~s}, 6 \mathrm{H}), 3.78(\mathrm{~s}, 3 \mathrm{H}) .{ }^{13} \mathrm{C}$ NMR (101 MHz, chloroform-d) $\delta$ 153.95, 152.41, 107.55, 93.16, 61.18, 56.18.

6-Chloropyridin-3-ol (2l). Pale yellow crystal, yield 88\%. Mp: 154-156 ${ }^{\circ} \mathrm{C} .{ }^{1} \mathrm{H}$ NMR (500 MHz, chloroform-d) $\delta 8.59(\mathrm{~s}, 1 \mathrm{H})$, $7.83(\mathrm{~d}, J=8.3 \mathrm{~Hz}, 1 \mathrm{H}), 7.47$ (d, $J=8.2 \mathrm{~Hz}, 1 \mathrm{H}) .{ }^{13} \mathrm{C}$ NMR $(125$ $\mathrm{MHz}$, chloroform-d) $\delta 151.88,147.92,137.19,124.87$.

Oxydibenzene (4a). Colorless liquid, yield 75\%. ${ }^{1} \mathrm{H}$ NMR $(400$ $\mathrm{MHz}$, chloroform-d) $\delta$ 7.41-7.28 (m, 4H), 7.14-7.06 (m, 2H), 7.05-6.95 (m, 4H). ${ }^{13} \mathrm{C}$ NMR (101 MHz, chloroform-d) $\delta$ 157.43, 129.87, 123.36, 119.04 .

4,4'-Oxybis(methylbenzene) (4b). Colorless liquid, yield $64 \%$. ${ }^{1} \mathrm{H}$ NMR (400 MHz, chloroform-d) $\delta 7.12(\mathrm{~d}, J=8.4 \mathrm{~Hz}, 4 \mathrm{H}), 6.89$ $(\mathrm{d}, J=8.5 \mathrm{~Hz}, 4 \mathrm{H}), 2.33$ (s, 6H). ${ }^{13} \mathrm{C} \mathrm{NMR}(101 \mathrm{MHz}$, chloroformd) $\delta 155.49,132.59,130.28,118.75,20.80$.

4,4'-Oxybis(fluorobenzene) (4c). Pale yellow liquid, yield $58 \%$. ${ }^{1} \mathrm{H}$ NMR (400 MHz, chloroform-d) $\delta$ 7.08-6.98 (m, 4H), 6.94 (dq, $J=6.7,2.8,2.2 \mathrm{~Hz}, 4 \mathrm{H}) .{ }^{13} \mathrm{C}$ NMR $(101 \mathrm{MHz}$, chloroform-d) $\delta 160.10,157.70,153.53,120.11,120.03,116.59$, 116.36 .

4,4'-Oxybis(chlorobenzene) (4d). Pale yellow liquid, yield $56 \% .{ }^{1} \mathrm{H}$ NMR (400 MHz, chloroform-d) $\delta 7.30(\mathrm{~d}, J=9.0 \mathrm{~Hz}$, $4 \mathrm{H}), 6.93$ (d, $J=8.9 \mathrm{~Hz}, 4 \mathrm{H}) .{ }^{13} \mathrm{C}$ NMR (101 MHz, chloroform-d) $\delta 155.74,130.01,128.85,120.25$.
4,4'-Oxybis(bromobenzene) (4e). Pale yellow liquid, yield $59 \% .{ }^{1} \mathrm{H}$ NMR (500 MHz, chloroform-d) $\delta 7.44$ (d, $J=8.9 \mathrm{~Hz}$, $4 \mathrm{H}), 6.88$ (d, $J=8.9 \mathrm{~Hz}, 4 \mathrm{H}) .{ }^{13} \mathrm{C}$ NMR $(125 \mathrm{MHz}$, chloroform-d) $\delta$ 156.12, 132.96, 120.70, 116.31.

4,4'-Oxybis(methoxybenzene) (4f). White liquid, yield $45 \%$. ${ }^{1} \mathrm{H}$ NMR (400 MHz, chloroform-d) $\delta 6.93(\mathrm{~d}, J=9.1 \mathrm{~Hz}, 4 \mathrm{H}), 6.86$ $(\mathrm{d}, J=9.0 \mathrm{~Hz}, 4 \mathrm{H}), 3.79$ (s, 6H). ${ }^{13} \mathrm{C} \mathrm{NMR}(101 \mathrm{MHz}$, chloroformd) $\delta 155.50,151.76,119.67,114.92,55.81$.

4,4'-Oxybis((trifluoromethoxy)benzene) (4g). Brown liquid, yield $60 \%$. ${ }^{1} \mathrm{H}$ NMR (500 MHz, chloroform-d) $\delta 7.21$ (d, $J=$ $8.9 \mathrm{~Hz}, 4 \mathrm{H}), 7.02$ (d, $J=9.0 \mathrm{~Hz}, 4 \mathrm{H}) .{ }^{13} \mathrm{C} \mathrm{NMR}(126 \mathrm{MHz}$, chloroform-d) $\delta 155.63,145.15,128.79,123.07,121.80,121.66$, 120.14, 119.75 .

Chloro-4-phenoxybenzene $(4 \mathrm{~h})$. Colorless liquid, yield $46 \%$. ${ }^{1} \mathrm{H}$ NMR (500 MHz, chloroform-d) $\delta 7.36(\mathrm{t}, J=7.3 \mathrm{~Hz}, 2 \mathrm{H}), 7.30$ $(\mathrm{d}, J=7.4 \mathrm{~Hz}, 2 \mathrm{H}), 7.14(\mathrm{t}, J=7.3 \mathrm{~Hz}, 1 \mathrm{H}), 7.02(\mathrm{~d}, J=7.8 \mathrm{~Hz}$, 2H), 6.95 (d, $J=7.5 \mathrm{~Hz}, 2 \mathrm{H}) .{ }^{13} \mathrm{C}$ NMR (126 MHz, chloroform-d) $\delta$ 156.98, 156.07, 130.00, 129.83, 128.30, 123.76, 120.16, 119.06.

1-Bromo-4-(4-methoxyphenoxy)benzene (4i). Pale yellow liquid, yield 44\%. ${ }^{1} \mathrm{H}$ NMR (500 MHz, chloroform-d) $\delta 7.38$ (d, $J$ $=8.2 \mathrm{~Hz}, 2 \mathrm{H}), 6.97(\mathrm{~d}, J=8.5 \mathrm{~Hz}, 2 \mathrm{H}), 6.89(\mathrm{~d}, J=8.4 \mathrm{~Hz}, 2 \mathrm{H})$, $6.82(\mathrm{~d}, J=8.1 \mathrm{~Hz}, 2 \mathrm{H}), 3.81(\mathrm{~s}, 3 \mathrm{H}) .{ }^{13} \mathrm{C} \mathrm{NMR}(126 \mathrm{MHz}$, chloroform-d) $\delta$ 157.93, 156.32, 149.73, 132.63, 121.06, 119.30, 115.10, 114.84, 55.80.

\section{Conflicts of interest}

There are no conflicts to declare.

\section{Acknowledgements}

The work was supported by Special Innovation Project of Guangdong Education Department (Natural Science) (2017KTSCX107), Medical Scientific Research Foundation of Guangdong province (B2019004), Science and Technology Planning Project of Guangdong Province (2016A020215159, 2017ZC0199), Key Laboratory of New Drug Discovery and Evaluation of Ordinary Universities of Guangdong province (2017KSYS002), Guangzhou Key Laboratory of Construction and Application of New Drug Screening Model Systems (201805010006), Guangdong province Precise Medicine and Big Data Engineering Technology Research Center for Traditional Chinese Medicine, the Open Project Foundation of the Fujian Provincial Key Laboratory of Theoretical and Computational Chemistry (201502).

\section{Notes and references}

1 (a) Z. Rappoport, The Chemistry of Phenols, Wiley-VCH, Weinheim, 2003; (b) J. H. P. Tyman, Synthetic and Natural Phenols; Elsevier: Amsterdam, 1996.

2 (a) H.-J. Arpe, Industrial Organic Chemistry, Wiley-VCH, Weinheim, 5th edn, 2010, pp. 359-374; (b) L. Wu, R. Song, S. Luo and J. Li, Angew. Chem., Int. Ed., 2018, 57, 1330813312; (c) W. Liu and L. Ackermann, Org. Lett., 2013, 15, 3484-3486; (d) T. Imahori, T. Tokuda, T. Taguchi and H. Takahata, Org. Lett., 2012, 14, 1172-1175; (e) D. Hojo 
and K. Tanaka, Org. Lett., 2012, 14, 1492-1495; (f) A. Kinbara, T. Yamagishi, N. Hanzawa, E. Kawashima and H. Miyaoka, J. Org. Chem., 2012, 77, 8999-9005; ( $g$ ) X. Chen, J. Zhang, X. Fu, M. Antonietti and X. Wang, J. Am. Chem. Soc., 2009, 131, 11658-11659; (h) M. Wang, M. Liu, H. Li, Z. Zhao, X. Zhang and F. Wang, ACS Catal., 2018, 8, 6837-6843; (i) S. Ghosh, S. Acharyya, T. Kaneko, K. Higashi, Y. Yoshida, T. Sasaki and Y. Iwasawa, ACS Catal., 2018, 8, 11979-11986.

3 (a) S. Enthaler and A. Company, Chem. Soc. Rev., 2011, 40, 4912; (b) P. S. Fier and K. M. Maloney, Org. Lett., 2016, 18, 2244; (c) M. C. Willis, Angew. Chem., Int. Ed., 2007, 46, 3402; (d) D. Zhao, N. Wu, S. Zhang, P. Xi, X. Su, J. Lan and J. You, Angew. Chem., Int. Ed., 2009, 48, 8729-8732; (e) L. Yang, Z. Huang, G. Li, W. Zhang, R. Cao, C. Wang, J. Xiao and D. Xue, Angew. Chem., Int. Ed., 2018, 57, 19681972; (f) L. Jing, J. Wei, L. Zhou, Z. Huang, Z. Li and X. Zhou, Chem. Commun., 2010, 46, 4767; $(g)$ K. G. Thakur and G. Sekar, Chem. Commun., 2011, 47, 6692; (h) Y. Wang, C. Zhou and R. Wang, Green Chem., 2015, 17, 3910; (i) S. Xia, L. Gan, K. Wang, Z. Li and D. Ma, J. Am. Chem. Soc., 2016, 138, 13493; (j) Y. Ren, L. Cheng, X. Tian, S. Zhao, J. Wang and C. Hou, Tetrahedron Lett., 2010, 51, 43; $(k)$ K. W. Anderson, T. Ikawa, R. E. Tundel and S. L. Buchwald, J. Am. Chem. Soc., 2006, 128, 10694; $(l)$ P. S. Fier and K. M. Maloney, Angew. Chem., Int. Ed., 2017, 56, 4478.

4 (a) J. J. Molloy, T. A. Clohessy, C. Irving, N. A. Anderson, G. C. Lloyd-Jonesc and A. J. B. Watson, Chem. Sci., 2017, 8, 1551-1559; (b) G. S. Dorta, D. M. Monzon, F. P. Crisostomo, T. Martın, V. S. Martına and R. Carrillo, Chem. Commun., 2015, 51, 7027-7030; (c) C. Zhu, R. Wang and J. R. Falck, Org. Lett., 2012, 14, 3494-3497; (d) D.-S. Chen and J.-M. Huang, Synlett, 2013, 24, 499-501; (e) E. Saikia, S. J. Bora and B. Chetia, RSC Adv., 2015, 5, 102723-102726; (f) S. Gupta, P. Chaudhary, L. Seva, S. Sabiah and J. Kandasamy, RSC Adv., 2015, 5, 8913389138; (g) J. P. Lambooy, J. Am. Chem. Soc., 1950, 72, 53275328.

5 (a) M. Bielawski and B. Olofsson, Chem. Commun., 2007, 2521-2523; (b) M. Bielawski, D. Aili and B. Olofsson, J. Org. Chem., 2008, 73, 4602-4607.

6 (a) N. Purkait, G. Kervefors, E. Linde and B. Olofsson, Angew. Chem., Int. Ed., 2018, 57, 11427-11431; (b) K. Matsuzaki, K. Okuyama, E. Tokunaga, N. Saito, M. Shiro and
N. Shibata, Org. Lett., 2015, 17, 3038-3041; (c) P. Li, G. Cheng, H. Zhang, X. Xu, J. Gao and X. Cui, J. Org. Chem., 2014, 79, 8156-8162.

7 L. Chan, A. McNally, Q. Y. Toh, A. Mendoza and M. J. Gaunt, Chem. Sci., 2015, 6, 1277-1281.

8 T. B. Petersen, R. Khan and B. Olofsson, Org. Lett., 2011, 13, 3462-3465.

9 M. Reitti, R. Gurubrahamam, M. Walther, E. Lindstedt and B. Olofsson, Org. Lett., 2018, 20, 1785-1788.

10 (a) D. Zhu, Z. Wu, B. Luo, Y. Du, P. Liu, Y. Chen, Y. Hu, P. Huang and S. Wen, Org. Lett., 2018, 20, 4815-4818; (b) J. Li, Q. Xu, Z. Wang, Y. Li and L. Liu, ACS Omega, 2018, 3, 12923-12929.

11 (a) D. Shen, C. Saracini, Y. Lee, W. Sun, S. Fukuzumi and W. Nam, J. Am. Chem. Soc., 2016, 138, 15857-15860; (b) M. Zhang, M. deRespinis and H. Frei, Nat. Chem., 2014, 6, 362-367.

12 (a) K. C. Nicolaou, C. N. C. Boddy, S. Bräse and N. Winssinger, Angew. Chem., Int. Ed., 1999, 38, 2096-2152; (b) Y. C. Song, F. Y. Lin, F. L. Yin, M. Hensler, C. A. R. Poveda, D. Mukkamala, R. Cao, H. Wang, C. T. Morita and D. G. Pacanowska, J. Med. Chem., 2009, 52, 976-988; (c) V. Sandanayaka, B. Mamat, R. K. Mishra, J. Winger, M. Krohn, L.-M. Zhou, M. Keyvan, L. Enache, D. Sullins and E. Onua, J. Med. Chem., 2010, 53, 573-585; (d) C. C. Lee, M. K. Leung, P. Y. Lee, T. L. Chiu, J. H. Lee, C. Liu and P. T. Chou, Macromolecules, 2012, 45, 751-765; (e) M. Lee, M. Ikejiri, D. Klimpel, M. Toth, M. Espahbodi, D. Hesek, C. Forbes, M. Kumarasiri, B. C. Noll and M. Chang, ACS Med. Chem. Lett., 2012, 3, 490-495; (f) M. J. R. P. Queiroz, D. Peixoto, R. C. Calhelha, P. Soares, T. dos Santos, R. T. Lima, J. F. Campos, R. M. V. Abreu, I. C. F. R. Ferreira and M. H. Vasconcelos, Eur. J. Med. Chem., 2013, 69, 855-862.

13 (a) X. Huang, Q. Zhu and Y. Xu, Synth. Commun., 2001, 31, 2823; (b) M. K. Muthyala, S. Choudhary, K. Pandey, G. M. Shelke, M. Jha and A. Kumar, Eur. J. Org. Chem., 2014, 2365.

14 (a) C. J. Teskey, S. M. A. Sohel, D. L. Bunting, S. G. Modha and M. F. Greaney, Angew. Chem., Int. Ed., 2017, 56, 52635266; (b) S. G. Modha and M. F. Greaney, J. Am. Chem. Soc., 2015, 137, 1416-1419; (c) S. G. Modha, M. V. Popescu and M. F. Greaney, J. Org. Chem., 2017, 82, 11933-11938. 